\title{
Clustering of Fermi particles with arbitrary spin
}

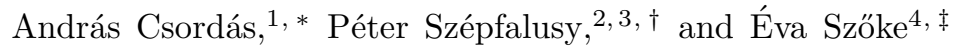 \\ ${ }^{1}$ Research Group for Statistical Physics of the Hungarian Academy of Sciences, \\ Pázmány P. Sétány 1/A, H-1117 Budapest, Hungary \\ ${ }^{2}$ Department of Physics of Complex Systems, Eötvös University, \\ Pázmány P. Sétány 1/A, H-1117 Budapest, Hungary \\ ${ }^{3}$ Research Institute for Solid State Physics and Optics, P. O. Box 49, H-1525 Budapest, Hungary \\ ${ }^{4}$ Budapest University Catholic Secondary School, Szabó Ilonka u. 2-4, H-1015 Budapest, Hungary
}

(Dated: September 12, 2018)

\begin{abstract}
A single $l$-shell model is investigated for a system of fermions of spin $s$ and an attractive $s$-wave, spin channel independent, interaction. The spectra and eigenvectors are determined exactly for different $l, s$ values and particle numbers $N$. As a generalization of Cooper pairing it is shown that when $N=\mu(2 s+1), \mu=1,2, \ldots, 2 l+1$, the ground state consists of clusters of $(2 s+1)$ particles. The relevance of the results for more general situations including the homogeneous system is briefly discussed.
\end{abstract}

PACS numbers: $32.80 . \mathrm{Pj}, 05.30 . \mathrm{Fk}, 74.20 . \mathrm{Fg}$

The experimental realizations of highly degenerate atomic Fermi gases confined in traps [1, 2, 3, 4, 5, 6, 6] have increased the activity in the theoretical investigation of such systems, which had already started earlier in view of the hope of future experiments. In particular many aspects of the possible transition to the superfluid phase have been studied in the case of the attractive interaction between the particles 8, 9, 10, 11, 12, 13, 14, 15, 16, 17, 18, 19, 20, 21, 22, 23, 24, 25, 26, 27. For instance, the critical temperature for the Cooper pairing of atoms with spin values higher than $1 / 2$ has been investigated [12, 17] based on the Gorkov-Melik-Barkhudarov approach to calculate corrections to the BCS theory due to induced interaction [28]. The result is that the critical temperature of the BCS theory is multiplied by $(4 e)^{\nu / 3-1}$, where $\nu$ denotes the number of fermion species $(\nu=2 s+1)$ 17]. Accordingly, if $s=1 / 2$ (the case treated by Gorkov-Melik-Barkhudarov) the critical temperature is decreasing, while it is increasing for $s \geq 3 / 2$. In the special case $s=1$ no such correction exists 12 .

The aim of the present paper is to propose and demonstrate that the superfluid ground state can be different from that given by the BCS pairing theory if the spin of the atoms $s>1 / 2$. Namely, we suggest that instead of Cooper pairs the particles can create clusters of zero total spin value containing $2 s+1$ particles. This can occur in optical traps where the spins of the particles can freely rotate (Alternatively, one can think about $2 s+1$ hyperfine states. In the following we use always the terminology of spin). We do not attempt to make any comparison with experimental results in this paper and choose the possible simplest model to make the presentation free from approximations. We hope that in future experiments the clustering effect can be revealed. We treat the case when the number of species $(2 s+1)$ is even. It is straightforward to extend our considerations for odd number of species. Note that in the spin-1 Fermi superfluid the pair correlation has been investigated in detail by Modawi and Leggett [12].

We assume that the trap potential is spherically symmetric. The investigation will be done at zero temperature and is restricted to the subspace of a given $l$-shell filled with particles of number $N(0<N<$ $(2 l+1)(2 s+1))$. The interaction between the particles in the open shell is described by a spin independent contact potential representing $s$-wave scattering with a negative scattering length:

$$
H_{i n t}=-\frac{\lambda}{2} \sum_{\substack{i, j=1 \\ i \neq j}}^{N} \delta\left(\mathbf{r}_{i}-\mathbf{r}_{j}\right) .
$$

The one particle normalized wave functions in the open shell are given by

$$
\Psi_{n, l, m_{l}, s, m_{s}}(r, \vartheta, \varphi, \sigma)=R_{n, l}(r) Y_{m_{l}}^{l}(\vartheta, \varphi) \chi_{m_{s}}^{s}(\sigma),
$$

Here $\sigma$ is a discrete spin variable, which can take $(2 s+1)$ different values. Spin eigenfunctions are orthonormalized according to

$$
\sum_{\sigma} \chi_{m_{s}}^{s}(\sigma) \chi_{m_{s}^{\prime}}^{s^{\prime}}(\sigma)=\delta_{s, s^{\prime}} \delta_{m_{s}, m_{s}^{\prime}}
$$

The functions (2) are eigenfunctions of the one-particle Hamiltonian, which contains besides the trap potential also the average field of the closed shells. The problem of diagonalizing the operator (11) can be solved exactly on the fixed basis (2).

Let us denote by $a_{m_{l}, m_{s}}$ the operator which annihilates a particle with quantum numbers $\left(n, l, m_{l}, s, m_{s}\right)$. In second quantization (11) can be written as

$$
\begin{aligned}
\hat{H}_{\text {int }} \equiv \hat{h} E_{0} / \pi= & -\frac{E_{0}}{2} \sum_{m_{1}, m_{2}, m_{3}, m_{4}} \sum_{\nu_{1}, \nu_{2}} f_{m_{1}, m_{2}, m_{3}, m_{4}} \\
& \times a_{m_{1}, \nu_{1}}^{+} a_{m_{2}, \nu_{2}}^{+} a_{m_{4}, \nu_{2}} a_{m_{3}, \nu_{1}}
\end{aligned}
$$


where $E_{0}$ is the characteristic energy

$$
E_{0}=\lambda \int_{0}^{\infty}\left|R_{n, l}(r)\right|^{4} r^{2} d r
$$

and the coefficient $f$ is given in terms of the Wigner- $3 j$ symbols.

The Hamiltonian (4) can be converted into

$$
\hat{h}=\frac{[l]}{8} \hat{N}-\frac{[l]^{2}}{8} \sum_{\substack{L=0 \\
L: \text { even }}}^{2 l}\left(\begin{array}{ccc}
l & l & L \\
0 & 0 & 0
\end{array}\right)^{2} \hat{B}_{L}^{2}
$$

where the abbreviation $[a] \equiv(2 a+1)$ is used and the scalar "square"

$$
\hat{B}_{L}^{2}=\sum_{M=-L}^{L}(-1)^{L-M} \hat{B}_{L, M} \hat{B}_{L,-M}
$$

of the irreducible tensor operators

$$
\begin{aligned}
\hat{B}_{L, M}=\sum_{m=-l}^{l} \sum_{\nu=-s}^{s} & (-1)^{l-m} \sqrt{[L]}\left(\begin{array}{ccc}
l & l & L \\
m & M-m & -M
\end{array}\right) \\
& \times a_{m, \nu}^{+} a_{m-M, \nu}
\end{aligned}
$$

has been introduced. $\hat{N}$ stands for the particle number operator. We note that in cases of $l=1$ and $l=2$ the Hamiltonian can be expressed in terms of the operators $\hat{N}, C_{2}(S U(3)), C_{2}(S O(3))$ and $\hat{N}$, $C_{2}(S U(5)), C_{2}(S O(5))$, respectively, where $C_{2}(G)$ denotes the quadratic Casimir operator of the group $G$ 29].

We have calculated the full exact spectra and the eigenvectors of $\hat{h}$ for $s=1 / 2, l=1,2,3$ and for $s=3 / 2$, $l=1,2$ using some Mathematica and Fortran programs. Unfortunatelly, the size of the Hilbert-space (in the $L_{z}=0 S_{z}=0$ or $S_{z}=1 / 2$ subspace too) grows drastically. For higher $s$ we also have performed calculations for $l=1, s=5 / 2,7 / 2$ in the $L=0$ subspace for even $N$. The Young tableau technique has also been used to analyse the model. The details will be published elsewhere 29]. Results for the ground state energies up to the half filled shell can be found in Tables [I and III Above the half filling energies and eigenvectors can be obtained by particle $\leftrightarrow$ hole transformation. If one exact eigenstate has $N$ particle in the $l, s$-shell with energy $E(N)$ then there is an other exact eigenstate for particle number $N^{\prime}=N_{t}-N\left(N_{t} \equiv(2 s+1)(2 l+1)\right)$ with energy

$$
E\left(N_{t}-N\right)=E(N)+(2 l+1) s\left(N_{t} / 4-N / 2\right), \quad N \leq N_{t} / 2
$$

It is enlightening to discuss the structure of the ground state for even number of particles in coordinate representation as well. Let us start with the two particle states. The wave function is as follows:

$$
\psi(1,2)=\varphi\left(\mathbf{r}_{1}, \mathbf{r}_{2}\right)^{2 S+1} \chi\left(\sigma_{1}, \sigma_{2}\right)
$$

The function $\varphi$ is symmetric and is the eigenfunction of the angular momentum operator with $L=0$, while the spin functions are antisymmetric. The latter is ensured if the total spin $S$ takes the values $S=0,2, \ldots, 2 s-1$, which makes the ground state $2 s$ times degenerate. The degeneracy is lifted and a spin multiplet arises, if the $s$-wave scattering lengths were different in different spin channels. In case of $s=1 / 2$ it is found that the exact ground states are "pair states" for $N=2,4, \ldots, 2(2 l+1)$, i.e., their wave functions are the antisymmetrized products of singlet pair wave functions

$$
\Psi(1,2, \ldots, N)=\hat{A} \prod_{i=1}^{N / 2} \psi(2 i-1,2 i)
$$

The operator $\hat{A}$ stands for antisymmetrization. The situation is different when $s>1 / 2$. Namely, instead of pair states we find that $2 s+1$ particle clusters are created when $N=\mu(2 s+1), \mu=1,2, \ldots, 2 l+1$. The wave function reads as

$$
\Psi(1,2, \ldots, N)=\hat{A} \prod_{i}^{\prime} \begin{aligned}
& \varphi\left(\mathbf{r}_{i}, \mathbf{r}_{i+1}, \ldots, \mathbf{r}_{i+2 s}\right) \\
& { }^{1} \chi\left(\sigma_{i}, \sigma_{i+1}, \ldots, \sigma_{i+2 s}\right)
\end{aligned}
$$

The prime on the product sign in Eq. (11) means that $i$ extends to the values as follows:

$$
i=(2 s+1) k+1, \quad k=0,1, \ldots, \mu-1
$$

${ }^{1} \chi$ denotes the singlet spin function representing the Slater determinant of the $(2 \mathrm{~s}+1)$ one particle spin funtions $\chi_{m_{s}}$

$$
\begin{aligned}
& { }^{1} \chi\left(\sigma_{1}, \sigma_{2}, \ldots, \sigma_{2 s+1}\right)=\frac{1}{\sqrt{(2 s+1) !}} \\
& \times\left|\begin{array}{cccc}
\chi_{-s}\left(\sigma_{1}\right) & \chi_{-s}\left(\sigma_{2}\right) & \ldots & \chi_{-s}\left(\sigma_{2 s+1}\right) \\
\chi_{-s+1}\left(\sigma_{1}\right) & \chi_{-s+1}\left(\sigma_{2}\right) & \ldots & \chi_{-s+1}\left(\sigma_{2 s+1}\right) \\
\vdots & \vdots & \ddots & \vdots \\
\chi_{s}\left(\sigma_{1}\right) & \chi_{s}\left(\sigma_{2}\right) & \ldots & \chi_{s}\left(\sigma_{2 s+1}\right)
\end{array}\right|
\end{aligned}
$$

In Eq. (11) $\varphi$ is a completely symmetric function for the exchange of any pair of particles. Furthermore, it is an eigenfunction of the orbital angular momentum operator of the $2 s+1$ particles with eigenvalue zero.

Eq. (11) means that the states can be created by repeated application of a creation operator to the vacuum $\left(\hat{Q}_{2 s+1}^{(l)}\right)^{\mu}|0\rangle$. We have derived this operator, but we do not present here in general form. For $s=1 / 2$ the operator $\hat{Q}_{2}^{(l)}$ is the pair creation operator

$$
Q_{2}^{(l)}=\frac{1}{2} \sum_{m=-l}^{l} \sum_{\nu=-1 / 2}^{1 / 2}(-1)^{(l-m+1 / 2-\nu)} a_{m, \nu}^{+} a_{-m,-\nu}^{+}
$$




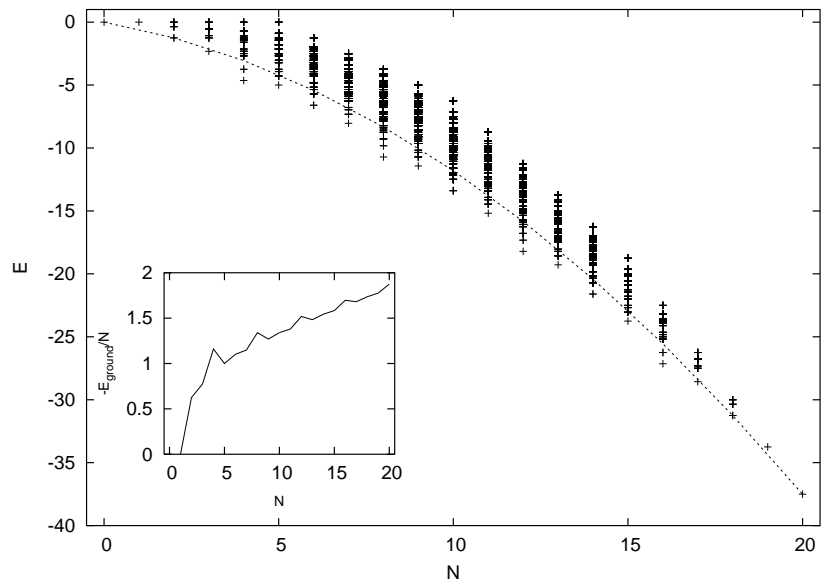

FIG. 1: The energy levels of the dimensionless Hamilton operator $\hat{h}$ in case of $l=2, s=3 / 2$. The dotted line (drawn everywhere to lead the eye) at even $N$ gives the average energy calculated using the pair wave function. The insertion shows the binding energy per particle as a function of the number of particles.

and for the ground state energy the simple relationship is obtained for $s=1 / 2$ :

$$
E_{0}=\frac{2 l+1}{8 \pi} N, \quad N(\text { even }) \leq 2(2 l+1)
$$

Furthermore, for $N=2$ Eq. (15) gives also the ground state energy for any $s$ values.

One can show that in the single $l$-shell model the cluster wave function $\varphi$ in Eq. (11) has the structure

$$
\varphi\left(\mathbf{r}_{1}, \ldots, \mathbf{r}_{2 s+1}\right)=\hat{S} \prod_{j=1}^{s+1 / 2} \varphi\left(\mathbf{r}_{2 j-1}, \mathbf{r}_{2 j}\right),
$$

where $\hat{S}$ denotes the operator for symmetrization and $\varphi\left(\mathbf{r}_{1}, \mathbf{r}_{2}\right)$ is the function defined in Eq. (9). It can be expressed in terms of the one particle wave functions:

$$
\begin{aligned}
\varphi\left(\mathbf{r}_{1}, \mathbf{r}_{2}\right)= & R_{n, 1}\left(r_{1}\right) R_{n, 2}\left(r_{2}\right) \\
& \times \sum_{m=-l}^{l}(-1)^{l-m} Y_{m}^{l}\left(\Omega_{1}\right) Y_{-m}^{l}\left(\Omega_{2}\right) .
\end{aligned}
$$

In Fig. 1 besides the exact levels for $l=2, s=3 / 2$ the expectation value of the Hamiltonian $\hat{h}$, Eq. (4), with wave function (10) is also shown. As can be seen it provides the exact ground state energy only for $N=$ $2,(2 s+1)(2 l+1)-2,(2 s+1)(2 l+1)$. In the other cases the energy obtained with the help of (10) lies considerably higher than the ground state energy. This is especially true when $N=\mu(2 s+1)$, and the wave function (11) applies. The binding energy per particle has maximum at these particle numbers.

For understanding the origin of the ground state wave function (11) one has to realize that the relevant group of the model is $S U(2 l+1) \otimes S U(2 s+1)$. With the usual decomposition one gets states characterized by quantum numbers specifying the irreducible representations of $S U(2 l+1) \supset S O(2 l+1) \supset S O(3)$ and similarly for $S U(2 s+1)$, where the relevant quantum number is the total spin related to the irreducible representations of $S U(2) \subset S U(2 s+1)$. The seniority is associated with $S O(2 l+1)$, while the orbital angular momentum $L$ with $S O(3)$. The validity of the cluster wave function $\Psi$ can be best understood by studying first the structure of its spin function. For $\mu=1$ the spin function is obviously invariant under the group $S U(2 s+1)$. It means that the generators of $S U(2 s+1)$ commute with the operator $\hat{Q}_{2 s+1}^{(l)}$ introduced in the text above (14). Since $\Psi$ is obtained by repeated application of $\hat{Q}_{2 s+1}^{(l)}$ it follows that $\Psi$ is also invariant under $S U(2 s+1)$ (consequently $S=0$ ). The corresponding Young tableau consists of $\mu$ columns of length $(2 s+1)$, which is known to specify a one dimensional irreducible representation of $S U(2 s+1)$ (see e.g. [30]). The Pauli principle to be fulfilled by $\Psi$ requires that the corresponding Young tableau of $S U(2 l+1)$ must consist of $\mu$ rows, each of length $(2 s+1)$. It contains for each $\mu$ a one dimensional representation of $S O(2 l+1)$, which leads to $L=0$. The state is unambigously determined by choosing this representation of $S O(2 l+1)$. We emphasize that the symmetry of $\Psi$ is higher than just the rotational invariance in the coordinate space and in the spin space. As a matter of fact the symmetry is the highest possible for particle numbers $N=\mu(2 s+1)$.

In summary, our results are in harmony with the expectation that the ground state of a system of Fermi particles with attractive interaction is the possible most symmetric one with respect to the exchange of the space coordinates of the particles. This feature manifests itself spectacularly, when the number of particles is the multiple of the number of species $2 s+1$. Though in this paper the single $l$-shell model has been treated one can presume the validity of the conclusions more generally.

As a generalization one can take into account more than one shell in constructing the cluster wave function $\varphi$ (configuration interaction). Note that the totally antisymmetric spin functions ${ }^{1} \chi$, Eq. (13), would not change. Another strategy would be to treat first the homogeneous system and apply a local density approximation for the trapped gas. The problem of the ground state of the homogeneous system is, of course, interesting in itself. In this case $\varphi$ is translationally invariant. When $s=1 / 2$ the wave function $\Psi$, Eq. (11), which coincides now with Eq. (10) is the well known expression of the BCS ground state projected onto the $N$ particle state and written in coordinate representation (see e.g. 31]). For the spin-1 Fermi gas, introduced by Modawi and Leggett [12], we predict a ground state containing three particles, clusters of zero spin and with a symmetric $\varphi\left(\mathbf{r}_{1}, \mathbf{r}_{2}, \mathbf{r}_{3}\right)$ function in the expression (111). 


\begin{tabular}{lccccccc}
\hline $\mathrm{N}$ & 2 & 4 & 6 & 8 & 10 & 12 & 14 \\
\hline \hline$s=1 / 2$ & $-3 / 4$ & & & & & & \\
$s=3 / 2$ & $-3 / 4$ & $-33 / 10$ & $-93 / 20$ & & & & \\
$s=5 / 2$ & $-3 / 4$ & $-33 / 10$ & $-153 / 20$ & $-48 / 5$ & & & \\
$s=7 / 2$ & $-3 / 4$ & $-33 / 10$ & $-153 / 20$ & $-69 / 5$ & $-327 / 20$ & $-207 / 10$ & \\
$s=9 / 2$ & $-3 / 4$ & $-33 / 10$ & $-153 / 20$ & $-69 / 5$ & $-87 / 4$ & $-249 / 10$ & $-597 / 20$ \\
\hline
\end{tabular}

TABLE I: Ground state energies of the dimensionless Hamilton operator $\hat{h}$ for $l=1$ and for $N=0,2, \ldots, 3(2 s+1) / 2$.

\begin{tabular}{cccccc}
\hline $\mathrm{N}$ & 2 & 4 & 6 & 8 & 10 \\
\hline \hline$s=1 / 2$ & $-5 / 4$ & $-5 / 2$ & & & \\
$s=3 / 2$ & $-5 / 4$ & $-65 / 14$ & $-185 / 28$ & $-75 / 7$ & $-375 / 28$ \\
\hline
\end{tabular}

TABLE II: Ground state energies of the dimensionless Hamilton operator $\hat{h}$ for $l=2$ and for $N=0,2, \ldots, 5(2 s+1) / 2$.

As an example let us assume that four particles with spin $3 / 2$ are put outside the Fermi sea of noninteracting particles as a generalization of the original Cooper problem for two particles (see for its discussion 31]). One has to emphasize that even that part of the wave function, which depends on the space coordinates does not have the "pair structure" (16) in general, it is valid only within the single $l$-shell model. One can use (16) as an ansatz, however, in a homogeneous system and compare it with (10) for the ground state. Now $\varphi\left(\mathbf{r}_{1}, \mathbf{r}_{2}\right)$ is specified by the requirement that it should satisfy the equation for a bound Cooper pair (because of the $\delta$ function nature of the two particle interaction one needs the usual regularization (see e.g. 11])). The result of a simple, but somewhat lengthy calculation leads to:

$E_{\text {pairs }}-E_{4 c l u s t e r}=\frac{5 \lambda}{V}\left[1+\frac{\varphi(\mathbf{0}) \int d^{3} r \varphi(\mathbf{r}) C(\mathbf{r})}{C^{2}(\mathbf{0})}\right]+O\left(\frac{1}{V^{2}}\right)$

where $E_{\text {pairs }}$ and $E_{4 c l u s t e r}$ are the expectation values of the Hamiltonian with the wave functions of form (10) and (1116) respectively, using the interaction (11). $V$ denotes the volume of the system. It has been used that $\varphi\left(\mathbf{r}_{1}, \mathbf{r}_{2}\right)=\varphi\left(\left|\mathbf{r}_{1}-\mathbf{r}_{2}\right|\right)$ can be taken real. The function $C(\mathbf{r})$ is defined as $C\left(\mathbf{r}_{1}-\mathbf{r}_{2}\right)=\int d^{3} r_{3} \varphi\left(\left|\mathbf{r}_{1}-\mathbf{r}_{3}\right|\right) \varphi\left(\mid \mathbf{r}_{3}-\right.$ $\left.\mathbf{r}_{2} \mid\right)$. As expected the difference (18) is of $O(1 / V)$. Since $\varphi$ and $C$ are dominantly positive the result shows that the wave function (11) is energetically favored due to the fact that (11) is "more symmetric" in space coordinates than (10). To get an energy gain of $O(1)$ a more accurate ground state wave function is needed, which can account for a possible four particle bound state. To find it goes beyond the scope of the present paper and is a part of our planned investigations.

The present work has been partially supported by the Hungarian Research National Foundation under Grant
Nos. OTKA T029552 and T038202.

* Electronic address: csordas@tristan.elte.hu

† Electronic address: psz@galahad.elte.hu

¥ Electronic address: szoke@ludens.elte.hu

[1] F. S. Cataliotti et al., Phys. Rev. A57, 1136 (1998).

[2] B. DeMarco and D. S. Jin, Science 285, 1703 (1999).

[3] A. G. Truscott et al., Science 291, 2570 (2001).

[4] F. Schreck et al., Phys. Rev. A 64, 011402(R) (2001).

[5] B. DeMarco, S. B. Papp, and D. S. Jin, Phys. Rev. Lett. 86, 5409 (2001).

[6] K. M. O'hara et al., Science 298, 2179 (2002).

[7] S. R. Granade, M. E. Gehm, K. M. O'Hara, and J. E. Thomas, Phys. Rev. Lett. 88, 120405 (2002).

[8] A. J. Leggett, Journal de Physique, Colloque C7, 41, 19 (1980).

[9] M. Yu. Kagan JETP Lett. 64, 301 (1996).

[10] H. T. C. Stoof, M. Houbiers, C. A. Sackett, and R. G. Hulet, Phys. Rev. Lett. 76, 10 (1996).

[11] M. Houbiers et al., Phys. Rev. A56, 4864 (1997)

[12] A. G. K. Modawi and A. J. Leggett, J. Low Temp. Phys. 109, 625 (1997).

[13] M. A. Baranov and D. S. Petrov, Phys. Rev. A58, R801 (1998).

[14] M. Houbiers and H. T. C. Stoof, Phys. Rev. A59, 1556 (1999).

[15] G. M. Bruun, Y. Castin, R. Dum, and K. Burnett, Eur. Phys. J. D7, 433 (1999).

[16] M. Mackie, E. Timmermans, R. Côté, and J. Javanainen, Optics Express 8, 118 (2000).

[17] H. Heiselberg, C. J. Pethick, H. Smith, and L. Viverit, Phys. Rev. Lett 85, 2418 (2000)

[18] M. Holland, S. J. J. M. F. Kokkelmans, M. L. Chiofalo, and R. Walser, Phys. Rev. Lett. 87, 120406 (2001).

[19] L. Viverit, S. Giorgini, L. P. Pitaevskii, S. Stringari, Phys. Rev. A 63, 033603 (2001).

[20] H. Heiselberg, Phys. Rev. A 63, 043606 (2001).

[21] A. Bulgac and Y. Yu, Phys. Rev. Lett. 88, 042504 (2002).

[22] M. L. Chiofalo, S. J. J. M. F. Kokkelmans, J. N. Milstein, and M. J. Holland, Phys. Rev. Lett. 88, 090402 (2002).

[23] H. Heiselberg and B. Mottelson, Phys. Rev. Lett. 88, 190401 (2002).

[24] G. M. Bruun and H. Heiselberg, Phys. Rev. A 65, 053407 (2002).

[25] G. M. Bruun, Phys. Rev. A 66, 041602(R) (2002).

[26] D. S. Petrov, M. A. Baranov and G. V. Shlyapnikov, Phys. Rev. A67, 031601(R) (2003).

[27] M. Grasso, M. Urban, cond-mat/0305588

[28] L. P. Gorkov and T. K. Melik-Barkhudarov, Sov. Phys. JETP 13, 1018 (1961).

[29] É. Szőke, A. Csordás, P. Szépfalusy to be published.

[30] M. Hamermesh, Group Theory and its Application to Physical Problems, (Addison-Wesley Publishing Company Inc., Reading, Massachusetts, 1964).

[31] J. R. Schrieffer, Theory of Superconductivity, Revised Printing, Advanced Book Program, (Westview Press, Boulder Colorado, 1999). 